Gynecologic and

Obstetric Investigation
Gynecol Obstet Invest 2011;72:10-14

DOI: $\underline{10.1159 / 000321390}$
Received: March 31, 2010

Accepted after revision: September 22, 2010

Published online: January 25, 2011

\title{
Comparison of the Efficacy of Three Progestins in the Treatment of Simple Endometrial Hyperplasia without Atypia
}

\author{
Ozlem Ozdegirmenci Fulya Kayikcioglu Ulku Bozkurt Mehmet Akif Akgul \\ Ali Haberal \\ Gynecology Clinic, Etlik Zubeyde Hanim Women's Health Teaching and Research Hospital, Ankara, Turkey
}

\section{Key Words}

Simple endometrial hyperplasia - Progestin • Remission •

Resolution

\begin{abstract}
Aim: To evaluate the treatment of endometrial hyperplasia (EH) with different progestins. Methods: Eighty-two women with simple EH without atypia were included. Patients were offered oral progestagens and were randomized to one of three options for 3 months: medroxyprogesterone acetate (MPA, 10 mg/day), lynestrenol (LYN, 15 mg/day) and norethisterone (NET, $15 \mathrm{mg} /$ day) for 10 days per cycle. Patients were reevaluated after treatment. Women diagnosed with proliferative and nonatypical $\mathrm{EH}$ at the second curettage were offered the same progestins for another 3 months. The primary outcome of the study was the proportion of women requiring further treatment. Results: Of the 82 women, 46 (56.1\%) received MPA (23.2\%), LYN (13.4\%) and NET (19.5\%) therapy for another 3 months at the end of the first 3 months of treatment. The patients receiving MPA showed resolution in $36.7 \%$ of the cases versus $37 \%$ in the NET group. The highest resolution rate (56\%) was observed in the LYN group, although there was no statistically significant difference between progestins regarding the proportion of women re-
\end{abstract}

quiring further treatment $\left(\chi^{2}=2.608 ; p=0.271\right)$. Conclusion: It seems that the efficacies of oral progestins are similar at these dosages in simple EH without atypia.

Copyright $\odot 2011$ S. Karger AG, Basel

\section{Introduction}

Endometrial hyperplasia (EH) is a common disease that involves proliferation of endometrial glands, which results in a greater than normal gland-to-stroma ratio together with varying degrees of architectural complexity and cytologic atypia. The number of new cases of EH per year in the Western world has been estimated at approximately 200,000 [1]. EH is most typically classified as simple or complex, with or without cytological atypia. The objectives of treating women with $\mathrm{EH}$ are to reduce abnormal bleeding and to prevent progression to endometrial cancer [2]. Simple EH often spontaneously regresses and rarely progresses to endometrial cancer [3]. Treatment options in simple $\mathrm{EH}$ include observation only, treatment with progestins at various dose regimens and surgery [4]. Progestin therapy is one of the effective therapies for simple EH without atypia. The female sex steroid estrogen stimulates endometrial proliferation in the nor-

\section{KARGER}

Fax +4161306 1234

E-Mail karger@karger.ch

www.karger.com
(C) 2011 S. Karger AG, Basel

0378-7346/11/0721-0010\$38.00/0

Accessible online at:

www.karger.com/goi
Ozlem Ozdegirmenci, MD

Etlik Zubeyde Hanim Women's Health Teaching and Research Hospital TR-06510 Ankara (Turkey)

Tel. +90 31222138 99, Fax +903123238191

E-Mail ozlemerman@gmail.com 
mal menstrual cycle, and a relative excess of this hormone compared with progesterone is thought to be the primary cause of EH $[4,5]$. Progestins exert antiestrogenic effects which lead to regression of $\mathrm{EH}$ [6].

Unfortunately, there is no consensus in the literature on the optimal dose and treatment time for EH. Furthermore, there are very few data on the type of progestin that is most effective, and the regimens advocated are essentially arbitrary $[2,7]$. Our primary goal was to assess the likelihood of histologic resolution under three different treatment options for simple $\mathrm{EH}$ without cytological atypia.

As far as we know, this is the first prospective followup study in which the effects of medroxyprogesterone acetate (MPA), lynestrenol (LYN) and norethisterone (NET) regimens are compared in simple EH without cytological atypia.

\section{Subjects and Methods}

An open, prospective randomized, single-center study was designed to evaluate the effectiveness of MPA, LYN and NET in premenopausal women with simple EH without cytological atypia. The study was approved by the local ethics committee of Etlik Zubeyde Hanim Women's Health Teaching and Research Hospital and informed consent was obtained from all the patients. All premenopausal women consecutively admitted to our department between January 1, 2006, and December 31, 2007, with histologically documented simple $\mathrm{EH}$ without atypia were included in the study. Premenopausal status was defined by serum folliclestimulating hormone measurement $(<20 \mathrm{IU} / \mathrm{l})$ and ongoing menstrual cycle for at least 6 months. All participants underwent transvaginal ultrasound scanning to exclude the presence of other pathologies. The initial diagnosis of $\mathrm{EH}$ was based on outpatient endometrial curettage material obtained using a Karman cannula $(7 \mathrm{~mm})$. Gynecologic pathologists assessed the histological samples and classified them using the 1994 World Health Organization classification of $\mathrm{EH}$ [8]. This classification is comprised of four categories: (1) simple EH without atypia, (2) complex EH without atypia, (3) simple atypical EH and (4) complex atypical $\mathrm{EH}$. Women with cytonuclear atypia were excluded from the study as were women treated with hormones in the previous 6 months and with associated pathologies of the endometrial cavity. Eligible patients were offered oral progestins and were randomized to one of the following three options for 3 months: oral MPA (10 mg/day), LYN (15 mg/day) and NET (15 mg/day) for 10 days between the 16th and 25th day of the menstrual cycle. Randomization was based on computer-generated codes. After 3 months of treatment, patients were reevaluated. Endometrial specimens were obtained between the 20th and 24th day of menstrual cycle by curettage using a Karman cannula. The curettage day was conditionally considered the first day of the artificially created menstrual cycle in all patients. The outcome was determined by comparing the diagnosis of the follow-up biopsy with the initial diagnosis. Response to treatment based on the last cu-

Comparison of Three Progestins in the

Treatment of Simple EH rettage specimen was defined as follows: (1) follow-up diagnosis of secretory-, inactive- or atrophic-pattern endometrium was classified as resolution; (2) follow-up diagnosis of proliferativepattern endometrium was classified as regression; (3) follow-up diagnosis of $\mathrm{EH}$ was classified as persistence if the specimen showed simple EH and (4) follow-up diagnosis of EH was classified as progression if the specimen showed complex $\mathrm{EH}$ [9].

The primary outcome of the study was the proportion of women requiring further treatment for 3 months according to the endometrial curettage specimen.

Women with resolution discontinued progestin therapy and were called for follow-up with transvaginal ultrasonography 3 months later. Women diagnosed with regression and persistence in the second curettage whilst using progestins were offered the same progestins for another 3 months and were then reevaluated with a third endometrial curettage at the end of the 6th month following treatment. Progestin treatment was abandoned and hysterectomy was recommended if there was no histological evidence of complete regression or if there was histological evidence of persistence of $\mathrm{EH}$ or progression of $\mathrm{EH}$ to atypia.

Power and Sample Size

The sample size of the study was calculated with $G^{*}$ Power (G*Power Ver. 3.00.10, Franz Faul, Universität Kiel, Germany) statistical packages. The required sample size for $90 \%$ power, $\alpha=$ 0.05 type I error, $\beta=0.10$ type II error and $f=0.40$ effect size was calculated as 84 including 28 patients in each group. Two additional cases were included in each group to allow for potential losses to follow-up, and the study was planned to be completed with a sample size of 90 .

\section{Statistical Analysis}

Statistical analysis was performed by using SPSS for Windows, Ver. 15.00 (SPSS Inc., Chicago, Ill., USA) statistical software. The Shapiro-Wilk test was used to test the normality of data. Descriptive data were expressed as means \pm standard deviation. Skewed data were shown as medians and interquartile ranges. Age, body mass index, and waist-to-hip ratios were compared by the oneway analysis of variance (one-way ANOVA). The gravidity and parity of the groups were compared by the Kruskal-Wallis nonparametric analysis of variance. The rates of response to treatments were calculated for the different types of progestins. Any differences between the rates of women requiring further treatment for 3 months were compared by the $\chi^{2}$ test. The levels of significance were set at $\mathrm{p} \leq 0.05$.

\section{Results}

Ninety patients (30 patients for each group) that met the inclusion criteria were enrolled into the study. Five patients in the LYN group and 3 patients in the NET group were lost to follow-up; the remaining 82 were eligible. The actual power of the study was $87 \%$ with this sample size. Thirty patients had been randomized to MPA, 25 to LYN and 27 to NET. All 82 women continued the study, and no discontinuation was observed second- 
ary to side effects of any drug. The side effect profile (acne in 2 , nausea in 2, weight gain in 3 patients) was comparable between the groups.

The age range was 30-57 years (mean $44.94 \pm 5.32$ ). The demographic characteristics of the groups are shown in table 1. The groups were comparable for age, gravidity, parity, body mass index and waist-to-hip ratio. Endometrial curettages were repeated in all patients at the end of the 3-month treatment. Contraception with condoms was advised during the treatment.

After the cyclic progestin treatment for 3 months, none of the cases progressed. We computed the proportion of women who responded to the 3-month treatment with the different progestins. Table 2 summarizes treatment outcome according to the results of endometrial histopathology. The patients receiving MPA showed resolution in $36.7 \%$ of the cases versus $37 \%$ in the NET group. The highest resolution rate (56\%) was observed in the LYN group, although there was no statistically significant difference between progestins regarding the rates of women requiring further treatment for 3 months $\left(\chi^{2}=2.608 ; \mathrm{p}=0.271\right)$. At the 6-month follow-up, no endometrial curettages were needed in this group. In order to evaluate the effect of age on the resolution rates, patients were divided into two groups: women $\leq 45$ years $(\mathrm{n}=40)$ and women $>45$ years $(\mathrm{n}=42)$. In patients $\leq 45$ years, the resolution rates were $41.2 \%(\mathrm{n}=17), 42.9 \%$ $(\mathrm{n}=7)$ and $43.8 \%(\mathrm{n}=16)$ under MPA, LYN and NET, respectively, whilst in patients $>45$ years, the resolution rates were $30.8 \%(\mathrm{n}=13), 61.1 \%(\mathrm{n}=18)$ and $27.3 \%(\mathrm{n}=$ 11). There were no statistically significant differences between the groups regarding the resolution rates with respect to age ( $\leq 45$ years; $\chi^{2}=0.023 ; p=0.989$ and $>45$ years; $\left.\chi^{2}=4.368 ; \mathrm{p}=0.113\right)$.

EH persisted after 3 months of treatment in 2 cases (1 in the MPA and 1 in the NET group). There was no persistence in the LYN group. The woman in the NET group was 43 years old and as she did not wish to retain her uterus, she underwent hysterectomy. The pathology of the material revealed simple EH without atypia. The other patient was 39 years old and preferred to receive MPA for another 3 months. The endometrial biopsy performed at the end of the 6th month showed an atrophic endometrium, and a levonorgestrel intrauterine system (LNGIUS) was inserted.

Of the 82 women, 46 (56.1\%) continued to receive MPA (23.2\%), LYN (13.4\%) and NET (19.5\%) therapy for another 3 months. There was no refusal of treatment in this group. Pathology of 1 patient in the LYN group showed complex EH without atypia and in 1 patient in the MPA
Table 1. Baseline demographic characteristics of the participants

\begin{tabular}{lllll}
\hline Characteristics & Group & $\begin{array}{l}\text { Mean } \pm \text { SD or } \\
\text { median (IQR) }\end{array}$ & Statistics & p \\
\hline Age & MPA & $\begin{array}{l}44.17 \pm 5.15 \\
46.88 \pm 5.11\end{array}$ & $\mathrm{~F}=2.489$ & 0.090 \\
& LYN & $\begin{array}{l}44.00 \pm 5.41 \\
\text { NET }\end{array}$ & & \\
\hline Gravidity & MPA & $3.0(2.0)$ & & \\
& LYN & $4.0(2.5)$ & $\chi^{2}=0.605$ & 0.739 \\
& NET & $4.0(2.25)$ & & \\
\hline Parity & MPA & $3.0(1.0)$ & & \\
& LYN & $3.0(2.0)$ & $\chi^{2}=3.335$ & 0.189 \\
& NET & $3.0(2.0)$ & & \\
\hline Body mass & MPA & $34.11 \pm 8.96$ & & \\
index & LYN & $31.88 \pm 5.99$ & $\mathrm{~F}=0.607$ & 0.548 \\
& NET & $32.49 \pm 5.76$ & & \\
\hline Waist-to-hip & MPA & $0.85 \pm 0.09$ & & \\
ratio & LYN & $0.86 \pm 0.06$ & $\mathrm{~F}=0.214$ & 0.808 \\
& NET & $0.85 \pm 0.05$ & &
\end{tabular}

$\mathrm{IQR}=$ Interquartile range.

${ }^{1} \mathrm{~F}=$ One-way ANOVA results; $\chi^{2}=$ Kruskal-Wallis results.

Table 2. Summary of outcomes at the end of the 3rd month of treatment with different progestins

\begin{tabular}{lllll}
\hline Progestin & Resolution & Regression & Persistence & Total \\
\hline MPA & $11(36.7)$ & $18(60.0)$ & $1(3.3)$ & $30(100)$ \\
LYN & $14(56.0)$ & $11(44.0)$ & 0 & $25(100)$ \\
NET & $10(37.0)$ & $16(59.3)$ & $1(3.7)$ & $27(100)$ \\
\hline
\end{tabular}

Data are expressed as numbers and percentages (in parentheses).

group pathology revealed simple $\mathrm{EH}$ after 6 months treatment. Both patients had proliferative endometria at the end of the 3rd month of treatment. They both underwent hysterectomy and final pathologies confirmed the diagnoses.

\section{Discussion}

The strength of our study resides in the fact that it was a randomized prospective trial and had high power to assess differences among the three different progestin regimens used. In this study, no statistically significant dif- 
ference was found between the different progestin regimens. Progestins are generally well tolerated and this was also the case in our current study, where none of the patients discontinued treatment [2]. This study is the only study in the English literature comparing the effectiveness of MPA, NET and LYN.

There are few studies in the literature investigating treatment modalities in EH. Ørbo et al. [7] compared the effects of LNG-IUS, MPA (10 mg) and no treatment in EH with and without atypia. They concluded that LNG-IUS was the optimal treatment for EH. They obtained 100\% effectiveness with the LNG-IUS as long as it remained in situ. They also reported that the outcome after an MPA regimen was comparable with observation only. The patients receiving oral progestin showed responses in 54\% of the cases, while in patients with observation only, spontaneous regression was seen in $50 \%$ of the cases after 6 months [7]. We inserted LNG-IUS in 1 woman whose $\mathrm{EH}$ responded to progestin treatment at the end of the $6 \mathrm{th}$ month. This treatment option has become more widespread during the last few years, but this option is not yet routinely used in our clinic. In a very recent cohort study, the likelihood of histologic persistence/progression of complex EH and atypical EH among women treated with progestin compared with those not treated was investigated. Women with complex atypical EH dispensed megestrol acetate had a $69 \%$ decreased risk of persistence/ progression compared with women who did not receive any progestin, and those dispensed MPA had a $49 \%$ decreased risk of persistence/progression [10]. The authors concluded that although progestin treatment of atypical $\mathrm{EH}$ was associated with a substantial increase in the likelihood of regression of the lesions, persistence/progression was nonetheless present in more than one quarter of treated women. Another study examined the likelihood of regression of $\mathrm{EH}$ in relation to progestin therapy. In that study, women with complex $\mathrm{EH}$ without atypia were treated with norethindrone acetate $(5 \mathrm{mg} /$ day) and MPA (10-20 mg/day) for 3-5 months, and a second biopsy was obtained; regression was evident in $61.5 \%$ [11]. Approximately, $60-70 \%$ of cases of complex EH have been reported to respond to progestin treatment $[12,13]$. In our study, we only enrolled patients with simple $\mathrm{EH}$ without atypia. The dosages and duration of progestin regimens except for LYN were chosen according to previous studies $[6,7]$. Bese et al. [6] evaluated the effect of 3 months of cyclic progesterone (NET, $15 \mathrm{mg} /$ day) treatment on proliferation and apoptosis in simple EH without atypia and reported that progesterone treatment reduced both proliferative and apoptotic activities in endometrial tissue of

Comparison of Three Progestins in the

Treatment of Simple EH these patients. We could not find any study investigating the LYN regimen in EH, but Dutta et al. [14] used LYN (15 mg/day) in women with abnormal uterine bleeding. So, we investigated the generally preferred dosages in our clinic. The resolution rates were similar in the MPA (36.7\%) and NET (37\%) groups. Although definitive conclusions could not be drawn, it would appear that LYN (15 mg/day) achieved a better resolution rate (56\%) compared with NET (15 mg/day) and MPA (10 mg/day). Additionally, it was interesting that LYN had a higher resolution rate (61.1\%) in patients $>45$ years. One reason for the higher response rate might be the older age of the LYN group. Although there were no statistically significant differences between the groups, the mean age of the LYN group was 46.88. A gradual decline in endogenous estrogen levels is observed as women get older. In a study on postmenopausal patients treated with oral progestins, a progesterone or LNG-IUS displayed a better response to treatment compared with premenopausal patients with complex atypical EH and well-differentiated endometrioid carcinoma [9]. Over $90 \%$ of the postmenopausal patients showed regression or complete resolution of their lesions as compared with less than half of the premenopausal patients. Further investigation is needed to determine whether LYN is a more effective treatment in older patients.

In the present study, it was observed that none of the cases of $\mathrm{EH}$ had progressed at the end of the 3-month follow-up. This interval was chosen because the majority of clinicians attempts treatment of EH for 8 weeks to 6 months and then reassesses pathology with a second biopsy [15]. EH was reversed in all patients except 2 and proliferation was decreased. This may be attributed to the antiproliferative effect of progesterone. Maximal glandular proliferative activity is observed during the follicular phase, and after the 3-day peak of luteinizing hormone, it decreases to its lowest levels $[6,16]$. This is why we performed the endometrial biopsies in the late luteal phase. Although the management of cases with proliferative endometrium at the 3-month follow-up is not clear, the treatment modality is generally left at the gynecologist's discretion. In these cases, continuing the treatment with the same or reduced dose can be advised, and at present this is our preferred clinical protocol [2]. Both estrogen and progesterone receptors are present at high levels in hyperplastic endometria [17]. One interesting point was whether response to progestins would be predicted by the receptor status of the hyperplastic endometrium. However, studies have repeatedly failed to show a relationship between receptor status and response to progestins [2, 
18]. Ferenczy and Gelfand [19] followed up the response of menopausal women with EH without and with cytologic atypia to oral MPA and reported a persistence rate of $14 \%$ in the patients without cytological atypia. In our study, the persistence rate was $7 \%$ after 3 months of treatment, indicating that the majority of women responded to progestins. However, 2 patients in the regression group continued progestin treatment for a further 3 months but, unfortunately, their third biopsies revealed $\mathrm{EH}$ again. This result might lead one to conclude that continuing the treatment with progestins and evaluating the result with an endometrial biopsy after treatment in cases of regression appears a logical management protocol.

It seems that the efficacies of these three progestins are similar at these dosages in simple EH without atypia. Our results do not allow any conclusion to be drawn as to the superiority or inferiority of one progestin over another. A limitation of our study could be the short follow-up time. Further prospective, randomized studies investigating the long-term effects of different progestins in simple EH are thus necessary.

\section{References}

1 Tropé C, Lindahl B: Premalignant lesions of the endometrium: clinical features and management; in Coppleson M (ed): Gynecologic Oncology, ed 2. New York, Churchill Livingstone, 1992, pp 747-751.

-2 Marsden DE, Hacker NF: Optimal management of endometrial hyperlasia. Best Pract Res Clin Obstet Gynaecol 2001;15:393-405.

3 Kurman RJ, Kaminski PF,Norris HJ: The behavior of endometrial hyperplasia. A longterm study of 'untreated' hyperplasia in 170 patients. Cancer 1985;56:403-412.

4 Haimovich S, Checa MA, Mancebo G, Fusté P, Carreras R: Treatment of endometrial hyperplasia without atypia in perimenopausal and postmenopausal women with a levonorgestrel intrauterine device. Menopause 2008;15:1002-1004.

-5 Anastasiadis PG, Skaphida PG, Koutlaki NG, Galazios GC, Tsikouras PN, Liberis VA: Descriptive epidemiology of endometrial hyperplasia in patients with abnormal uterine bleeding. Eur J Gynaecol Oncol 2000;21: 131-134.

-6 Bese T, Vural A, Ozturk M, Dagistanlı F, Demirkıran F, Tuncdemir M, Arvas M, Sanioglu C, Kosebay D: The effect of long-term use of progesterone therapy on proliferation and apoptosis in simple endometrial hyperplasia without atypia. Int J Gynecol Cancer 2006;16:809-813
7 Ørbo A, Arnes M, Hancke C, Vereide AB, Pettersen I, Larsen K: Treatment results of endometrial hyperplasia after prospective D-score classification: a follow-up study comparing effect of LNG-IUD and oral progestins versus observation only. Gynecol Oncol 2008;111:68-73.

8 Espindola D, Kennedy KA, Fischer EG: Management of abnormal uterine bleeding and the pathology of endometrial hyperplasia. Obstet Gynecol Clins N Am 2007;34:717737.

-9 Wheeler DT, Bristow RE, Kurman RJ: Histologic alterations in endometrial hyperplasia and well-differentiated carcinoma treated with progestins. Am J Surg Pathol 2007;31: 988-998.

10 Reed SD, Voigt LF, Newton KM, Garcia RH, Allison HK, Epplein M, Jordan D, Swisher E, Weiss NS: Progestin therapy of complex endometrial hyperplasia with and without atypia. Obstet Gynecol 2009;113:655-662.

- 11 Horn LC, Schnurrbusch U, Bilek K, Hentschel B, Einenkel J: Risk of progression in complex and atypical endometrial hyperplasia: clinicopathologic analysis in cases with and without progestogen treatment. Int J Gynecol Cancer 2004;14:348-353.

12 Vereide AB, Arnes M, Straume B, Maltau JM, Ørbo A: Nuclear morphometric changes and therapy monitoring in patients with endometrial hyperplasia: a study comparing effects of intrauterine levonorgestrel and systemic medroxyprogesterone. Gynecol Oncol 2003;91:526-533.
13 Wang S, Pudney J, Song J, Mor G, Schwartz $\mathrm{PE}$, Zheng W: Mechanisms involved in the evolution of progestin resistance in human endometrial hyperplasia - precursor of endometrial cancer. Gynecol Oncol 2003;88: 108-117.

14 Dutta A, Sen PN, Banerjee T: Effect of progesteron (lynoestrenol) on abnormal uterine bleeding. J Indian Med Assoc 1981;77:110113.

15 American College of Obstetricians and Gynecologists: ACOG practice bulletin clinical management guidelines for obstetrician-gynecologists, number 65, August 2005: management of endometrial cancer. Obstet Gynecol 2005; 106:413-425.

16 Ferenczy A, Bergeron C: Histology of the human endometrium: from birth to senescence. Ann NY Acad Sci 1991;622:6-27.

17 Contreras OO, Sananes CE: Hormonal receptors in endometrial neoplasias. Eur J Gynaecol Oncol 1988;9:396-398.

18 Lindahl B, Willen R: Steroid receptor concentrations as a prognostic factor in atypical endometrial hyperplasia. Anticancer Res 1998; 18:3793-3795.

19 Ferenczy A, Gelfand M: The biologic significance of cytologic atypia in progestin-treated endometrial hyperplasia. Am J Obstet Gynecol 1989;160:126-131. 